\section{SOI: 1.1/TAS DOI: 10.15863/TAS International Scientific Journal Theoretical \& Applied Science}

\author{
p-ISSN: 2308-4944 (print) e-ISSN: 2409-0085 (online) \\ Year: $2015 \quad$ Issue: $10 \quad$ Volume: 30
}

Published: $30.10 .2015 \quad$ http://T-Science.org

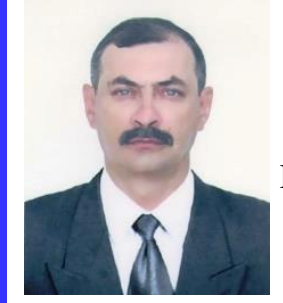

Andrey Stanislavovich Reshenkin

Candidate of Technical Sciences, head of department of

Don State Technical University, Russia reshenkin@list.ru

Dmitry Alexandrovich Tihomirov student of Don State Technical University, Russia

SECTION 7. Mechanics and machine construction.

\title{
HOW TO CONTROL THE STATE OF STRUCTURAL STRENGTH
}

Abstract: This paper presents a method for monitoring the strength of the power structures of ferromagnetic materials. In the method for design diagnosable affect alternating electromagnetic field, resulting in the measuring coil at the surface diagnosed structure induced electromotive force, the total response is called a magnetic noise is recorded instrumentation. The resulting signal is converted to a numerical value and compared with the basic signals. The technical result is to assess the current state of the strength of the power structures of ferromagnetic materials.

Key words: the magnetic noise, non-destructive testing, strength.

Language: Russian

Citation: Reshenkin AS, Tihomirov DA (2015) HOW TO CONTROL THE STATE OF STRUCTURAL STRENGTH. ISJ Theoretical \& Applied Science 10 (30): 45-49.

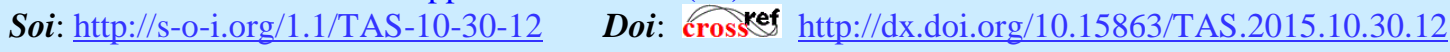

\section{СПОСОБ КОНТРОЛЯ СОСТОЯНИЯ ПРОЧНОСТИ СИЛОВЫХ КОНСТРУКЦИЙ}

Аннотация: В работе представлен способ контроля состояния прочности силовых конструкций из ферромагнитных материалов. При реализации способа на диагностируемую конструкцию воздействуют переменным электромагнитным полем, в результате чего в измерительной катушке, находящейся на поверхности диагностируемой конструкиии, индуцируется электродвижущая сила, суммарный отклик которой, называемый магнитным шумом, регистрируется измерительной аппаратурой. Полученный сигнал преобразуется $\kappa$ численному значению и сравнивается с базовыми сигналами. Техническим результатом является оценка текущего состояния прочности силовых конструкичй из ферромагнитных материалов.

Ключевые слова: магнитный шум, неразрушающий контроль, прочность.

Вопросы повышения достоверности и качества контроля состояния конструкций весьма актуальны и требуют постоянного совершенствования. В связи с этим работа относится к области диагностики состояния прочности силовых конструкций из ферромагнитных материалов и может найти применение при проведении технического освидетельствования силовых (несущих) конструкций.

Известно, что прочность конструкций обеспечивает требуемый уровень механических свойств материала, которые зависят от структуры и текстуры материала, количества и вида дефектов кристаллического строения, что формируется при различных технологических процессах изготовления конструкций и изделий [1].
При работе на конструкции воздействуют эксплуатационные нагрузки, приводящие как к износу, при действии сил трения, усталостному разрушению, вследствие циклического нагружения, так и изменению структуры и (или) текстуры материала, вследствие температурного воздействия или иных факторов. Кроме эксплуатационных нагрузок конструкции могут подвергаться нагрузкам, превышающим допустимые (при нарушениях правил эксплуатации), или, при аварийных ситуациях, воздействиям, не учитываемым при прочностном расчете (например, ударные воздействия, приводящие к образованию концентраторов напряжений, температурные воздействия при пожаре или локальном воздействии пламени, приводящие к текстурным и (или) структурным изменениям материала конструкций). Все изменения, происходящие в материале 
конструкций, влияют на механические свойства материала и тем самым на прочность конструкций.

В настоящее время существуют способы определения дефектов конструкций, в основе которых лежит фиксация аномалий магнитного поля при внешнем намагничивании [2]. Известны способы обнаружения механических повреждений конструкций на основе эффекта Холла [3].

Данные способы позволяют обнаружить дефекты и механические повреждения конструкций, но не пригодны для контроля состояния прочности при изменениях структуры и текстуры материала.

Так, например, способ диагностики элементов конструкции гребного винта [4], позволяет диагностировать процессы износа и усталости. Данный способ заключается в создании в контролируемых элементах конструкции вихревых токов, измерении сигналов ЭДС индукции вторичного переменного магнитного поля, сравнении формы и амплитуды сигналов с сигналами, соответствующими исправному состоянию конструкции.

К числу недостатков можно отнести следующее: данный способ позволяет обнаружить дефекты, связанные с износом и явления усталостного разрушения, т.е., как и способы, приведенные выше, не пригоден для контроля состояния прочности при изменениях структуры и (или) текстуры материала. Отклонение измеренных сигналов от сигналов, соответствующих исправному состоянию конструкции, позволяет установить факт ненахождения конструкции в исходном, т.е. полностью исправном состоянии, и не позволяет провести оценку текущего состояния прочности для установления возможности дальнейшей эксплуатации конструкции и (или) определения остаточного ресурса работы конструкции.

Известен магнитошумовой метод неразрушающего контроля, физическая сущность которого заключается в перемагничивании материала переменным магнитным полем, в результате которого в его поверхностном слое возбуждаются импульсные стохастические электромагнитные волны, обусловленные скачкообразным последовательным смещением стенок магнитных доменов. В измерительной катушке индуцируется электродвижущая сила, электромагнитные характеристики которой зависят от геометрических и электрофизических параметров материала. Суммарный отклик электродвижущей силы, регистрируемый измерительной аппаратурой, - это магнитный шум, параметры которого являются информативными [5].
Наиболее близким - по совокупности признаков - аналогом является способ магнитошумовой диагностики элементов кузова автомобилей, позволяющий оценивать техническое состояние элементов кузова автомобилей, выполненных из ферромагнетиков, при сравнении уровня магнитного шума диагностируемого элемента с эталонным сигналом, определяемым как среднее значение измеренного уровня магнитного шума бездефектных элементов кузовов автомобилей, или при сравнение с базой данных, содержащей информацию об уровне магнитного шума при наличии различных дефектов элементов кузова автомобиля [6].

К его недостатку можно отнести следующее: данный способ, позволяя обнаружить наличие различных скрытых лакокрасочным покрытием дефектов, не позволяет диагностировать состояние прочности конструкции.

Задача, на решение которой направлен предлагаемый способ, заключается в оценке текущего состояния прочности силовых конструкций из ферромагнитных материалов по результатам диагностики микроструктуры материала на основе применения магнитошумового метода неразрушающего контроля, позволяющей установление возможности дальнейшей эксплуатации, определение остаточного ресурса работы конструкций или показателей, характеризующих состояние прочности.

Техническим результатом предлагаемого изобретения является оценка текущего состояния прочности силовых конструкций из ферромагнитных материалов на основе применения магнитошумового метода неразрушающего контроля.

Технический результат достигается тем, что на диагностируемую конструкцию воздействуют переменным электромагнитным полем, в результате которого в поверхностном слое материала возбуждаются импульсные стохастические электромагнитные волны, обусловленные скачкообразным последовательным смещением стенок магнитных доменов. В измерительной катушке, находящейся на поверхности диагностируемой конструкции, индуцируется электродвижущая сила, суммарный отклик которой, называемый магнитным шумом, регистрируется измерительной аппаратурой. Полученный сигнал, характеризующийся временными и частотными характеристиками, по одному или нескольким параметрам (амплитуда, длительность, фаза, частота, спектральная плотность и др.) преобразуется к численному значению и сравнивается с базовыми сигналами. Базовые значения сигналов определяются экспериментально при точном соответствии 
режима воздействия

переменного электромагнитного поля на аналогичных образцах конструкций при воздействии всех возможных видов нагрузок до разрушения.

Полученные базовые значения сигналов формируют базу данных, в которой каждому значению, на основе экспериментально установленной взаимосвязи в виде функциональных зависимостей «состояние прочности - значение сигнала», присваивается состояние прочности, которое при этом может быть количественно выражено в требуемых для диагностируемой конструкции показателях (механическое свойство, наработка, максимально допустимая грузоподъемность, остаточный ресурс работы и др.).

Магнитошумовой способ контроля состояния прочности силовых конструкций из ферромагнитных материалов включает в себя следующую последовательность действий.

1. Силовые конструкции (изделия) подвергаются анализу с целью определения эксплуатационных нагрузок и возможных нагрузок при аварийных ситуациях.

2. Образцы различных частей силовых конструкций подвергаются экспериментальным исследованиям, в ходе которых на них воздействуют определенными в пункте 1 нагрузками, с одновременной магнитошумовой диагностикой и регистрацией сигнала магнитного шума, преобразованного к численному значению по одному или нескольким параметрам (амплитуда, длительность, фаза, частота, спектральная плотность и др.).

3. На основе экспериментов для всех видов нагрузок устанавливается взаимосвязь в виде «состояние прочности - значение сигнала», что и формирует базу данных. Состояние прочности может быть количественно выражено в требуемых для диагностируемой конструкции показателях (механическое свойство, наработка, максимально допустимая грузоподъемность, остаточный ресурс работы и др.). Для удобства пользования взаимосвязь «состояние прочности значение сигнала» может быть отражена графически.

4. При проведении технического освидетельствования с целью определения текущего состояния прочности силовые конструкции подвергаются магнитошумовой диагностике, при этом должны быть соблюдены режимные параметры магнитошумовой диагностики пункта 2.

5. Полученные сигналы сравниваются с сигналами базы данных и на основании этого устанавливается текущее состояние прочности силовой конструкции.

Возможность технической реализации данного способа доказана авторами при проведении экспериментальных исследований образцов из различных ферромагнитных сталей без термообработки и после различных видов термообработки при многоцикловом и малоцикловом нагружении до разрушения, температурных воздействиях с последующим испытанием на разрывной машине[7-10]. Ниже приведены результаты исследований циклической долговечности образцов из стали $40 \mathrm{X}$ прокат, свидетельствующие о технической реализуемости предлагаемого способа.

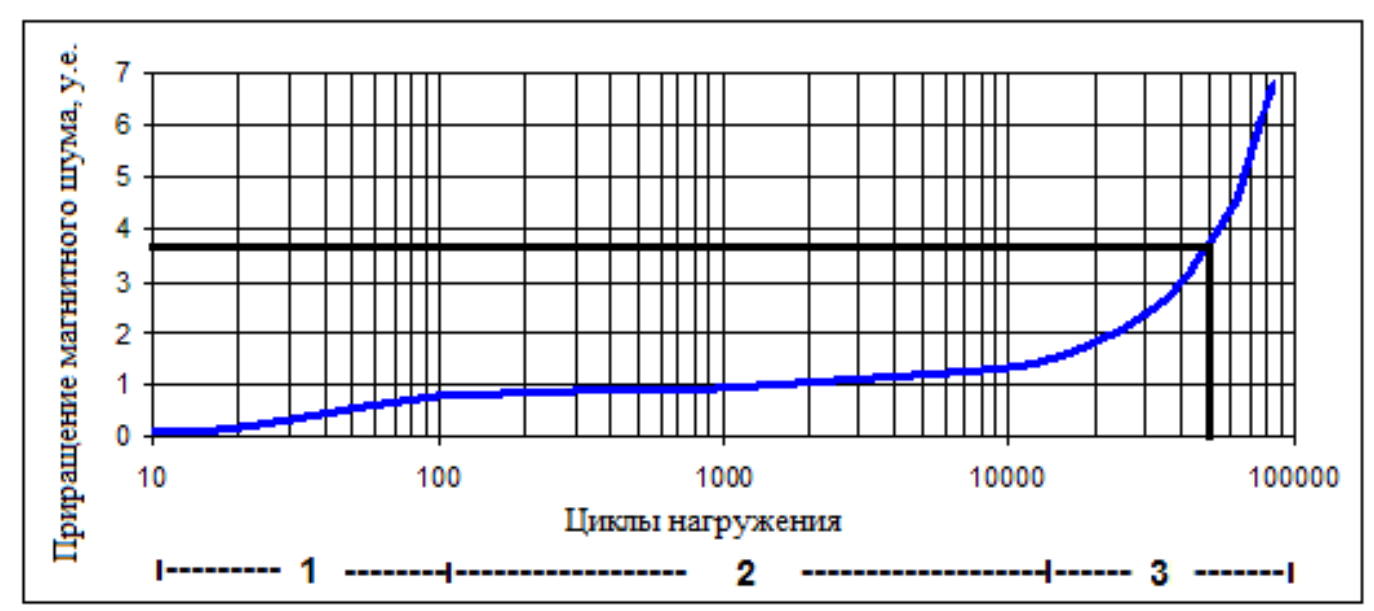

Рисунок 1 - К определению состояния прочности.

Образцы подвергались циклическому нагружению с периодическим осмотром и определением сигнала магнитного шума, в качестве которого в данном эксперименте была

ISPC Innovations in science, 
выбрана спектральная плотность, приведенная к условным единицам. На рисунке 1 представлена зависимость приращения сигнала магнитного шума от количества циклов нагружения с амплитудой деформации, обеспечивающей нагрузку, составляющую $80 \%$ от предела текучести, построенная в полулогарифмических координатах. За ноль принят исходный сигнал магнитного шума образцов до испытаний, который был постоянный по всей длине образцов, что обусловлено одинаковой структурой и текстурой материала, а также одинаковым количеством дефектов кристаллической структуры.

На полученной экспериментальной зависимости можно выделить три характерных участка. Первый участок характеризуется резким увеличением значений сигнала на первых 100 циклах, что по аналогии можно назвать приработкой, т.е. возникает некоторая поврежденность материала образца, незначительно влияющая на прочность. Второй участок характеризуется постепенным ростом сигнала, что свидетельствует о постепенном росте поврежденности материала. Третий участок характеризуется резким ростом сигнала. На третьем участке интенсивность накопления повреждений возрастает, достигая в конце критической величины, а механизм поврежденности переходит с микро- на макроуровень, который заканчивается образованием трещин на поверхности и разрушением при 82000 циклах нагружения.

Полученная зависимость является базовой для элементов конструкций, имеющих одинаковое с экспериментальными образцами сечение, выполненных из стали 40X прокат и подверженных циклическим нагрузкам, соответствующим экспериментальным. Для установления состояния прочности диагностируемой конструкции необходимо, при соблюдении режимных параметров магнитошумовой диагностики эксперимента, определить приращение сигнала магнитного шума, отложить на графике базовых сигналов и оценить текущее состояние прочности, определив (в данном случае) наработку и остаточный ресурс работы, выраженные в циклах нагружения. Например, при приращении сигнала магнитного шума 3,7 условных единицы (см. рисунок 1) наработка составляет около 40000 циклов, остаточный ресурс работы, определяемый как разница между количеством циклов до разрушения и наработкой, равен 52000 циклов.

Аналогичные испытания сварных швов, а также проведенные на разрывных машинах малоцикловые нагружения образцов и образцов после температурных воздействий (температурные воздействия приводили к текстурным и структурным изменениям материала образцов, что устанавливалось микроанализом) также доказали техническую реализуемость предлагаемого способа.

Предлагаемый способ позволяет провести оценку состояния прочности силовых конструкций из ферромагнитных материалов, на всех этапах жизненного цикла конструкций, и позволяет диагностировать снижение прочности конструкций, обусловленное наличием микротрещин, микроструктурными или текстурными изменениями материала до появления дефектов, наличие которых является причиной разрушения силовых конструкций.

Магнитошумовой способ контроля состояния прочности силовых конструкций из ферромагнитных материалов, основанный на использовании магнитошумового метода неразрушающего контроля, заключающийся в воздействии на диагностируемую конструкцию переменного электромагнитного поля, в результате которого в поверхностном слое материала возбуждаются импульсные стохастические электромагнитные волны, обусловленные скачкообразным последовательным смещением стенок магнитных доменов, а в измерительной катушке, находящейся на поверхности диагностируемой конструкции, индуцируется электродвижущая сила, суммарный отклик которой, называемый магнитным шумом, регистрируется измерительной аппаратурой, отличающийся тем, что полученный сигнал, характеризующийся временными и частотными характеристиками, по одному или нескольким параметрам (амплитуда, длительность, фаза, частота, спектральная плотность или другие параметры, полученные в результате обработки временных и частотных характеристик сигнала) преобразуется к численному значению и сравнивается с базовыми сигналами, которые определены экспериментально в том же порядке на аналогичных образцах конструкций при воздействии всех возможных видов нагрузок до разрушения и формируют базу данных, в которой каждому значению сигнала, на основе экспериментально установленной взаимосвязи в виде функциональных зависимостей «состояние прочности - значение сигнала», присваивается состояние прочности, которое при этом может быть количественно выражено в требуемых для диагностируемой конструкции показателях. 


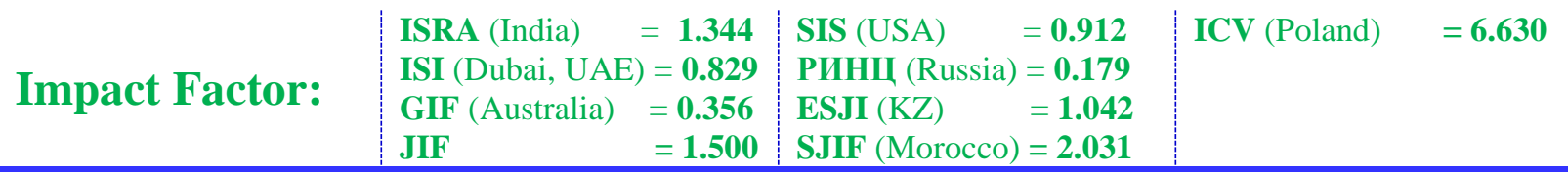

References:

1. Arzamasov BN, et al. (2004) Materialovedenie: - Moscow: MGTU im. Baumana, 2004. - 646 s.

2. (2002) Izmerenie, kontrol', kachestvo. Nerazrushayushii kontrol': Spravochnik. Moscow: IPK. Izdatel'stvo standartov, 2002. $708 \mathrm{p}$.

3. Kollakot R (1989) Diagnostika povrezhdenii: Per. s angl. - Moscow: Mir, 1989. - 512 p.

4. (1995) Patent na izobretenie RF №2032591, MPK6 V63N 1/02, 1995.

5. Vengrinovich VD, Bus'ko VN (1982) Magnitoshumovoi metod kontrolja himicheskogo sostava ferromagnitnyh splavov. // Defektoskopija. 1982 , №2.

6. Reshenkin AS, Tihomirov AG (2004) Ustroistvo dlja magnitoshumovoi diagnostiki yelementov kuzova avtomobilei. Svidetel'stvo na poleznuyu model' №38946, MPK7 G01N 27/72, 2004.
7. Reshenkin AS (2005) Magnitoshumovoi kontrol' ferromagnitnyh detalei //Problemy mashinostroenija i nadezhnosti mashin. 2005, №3, pp. 82-87.

8. Reshenkin AS (2005) Operativnaja ocenka tehnicheskogo sostojanija ferromagnitnyh konstrukcii // Kontrol'. Diagnostika. 2005, №4, pp. 22.

9. Reshenkin AS, Shapovalov AP (2007) Statisticheskaja ocenka parametrov mikrostruktury konstrukcionnyh stalei //Zagotovitel'nye proizvodstva v mashinostroenii. 2007, №1, pp.44-46.

10. Reshenkin AS, Goncharov AV, Tihomirov AG (2015) Obosnovanie primenenija SNraspredelenija dlja analiza parametrov mikrostruktury konstrukcionnyh stalei // Zagotovitel'nye proizvodstva $\mathrm{v}$ mashinostroenii. 2015, №1, pp.38-40. 